\title{
Taxonomy, at the click of a mouse
}

\section{Informatics and taxonomy are working together to achieve more than either could alone.}

Sir - We welcome your Opinion article "Genomics and taxonomy for all" (Nature $417,573 ; 2002)$ and H. C. J. Godfray's creative ideas for modernizing taxonomic practices in his Commentary "Challenges for taxonomy" (Nature 417, 17-19; 2002; see also News Feature pages 362-363). Taxonomy provides the reference system for all biology, so organizing, updating and streamlining the process of bringing taxonomic information onto every desktop at the click of a mouse are urgent priorities. The first substantial steps in this direction are already being taken, as mentioned by Godfray, and there is more to follow.

First, Species 2000 (www.sp2000.org) and the Integrated Taxonomic Information System (www.itis.usda.gov), two major players in creating an electronic global framework for taxonomy, joined forces last year in the Catalogue of Life consortium and are now making rapid progress with a catalogue of all known organisms. They have invited other organizations to join them in constructing a complete and freely available web-based synonymic index of species and associated data. The 2002 Catalogue of Life now lists 260,000 species $(860,000$ names, including synonyms and common names) on CD-ROM and on the Web. This programme is community-wide - the sources of this knowledge are the participating taxonomic database programmes embedded in the taxonomic profession and supported by systematics institutions, projects and individuals around the world.

Second, the Global Taxonomy Initiative (GTI) of the Convention on Biological Diversity (see decision VI/8 of COP 6 at www.biodiv.org/decisions) is building taxonomic capacity and making taxonomic information available to help implement the convention. A programme of work for the GTI was formally adopted at the Hague in April, providing political endorsement and a framework for national, regional and global projects to develop the knowledge base. Funding comes from various sources, including the Global Environment Facility.

Third, the Global Biodiversity Information Facility (GBIF; www.gbif.org) aims to develop an interoperable network of biodiversity databases and information technology tools for users to explore the world's vast quantities of biodiversity information for economic, environmental and social uses. GBIF is developing standards for interoperability, digitizing biodiversity data, helping to complete the Catalogue of Life, and improving global taxonomic and biodiversity informatics capacity. GBIF is also developing a portal to provide specialized search engines for accessing digitized, georeferenced specimen data from the world's herbaria, museums and other natural history collections. In this it is working closely with the Catalogue of Life consortium, GTI and other taxonomic organizations.

Fourth, we endorse Godfray's suggestion that species descriptions, images and a platform for publication and debate should be provided on the web. These online community-building features are not yet generally available for the Catalogue of Life, but individual contributing systems within Species 2000 are already experimenting in this area. ILDIS LegumeWeb (www.ildis.org) has the first 'community taxonomy' created and maintained by worldwide contributors to a global database. FishBase (www.fishbase. org) and AlgaeBase (www.algaebase.org) are experimenting with web-based facilities for users to offer images, data on new species and so on. At present none of these publishes the original diagnoses of new species, but the infrastructure that could provide this facility is already in place. GBIF is similarly planning a digital library of biodiversity literature and a 'Species Bank', to include the collaborative, online community-building features proposed by Godfray.

All these activities are in tune with Godfray's proposals and your Opinion article. The Catalogue of Life and the GBIF are each funded at about US $\$ 3$ million a year and we should see real progress over the next three years. Several projects under the aegis of the GTI have been funded, although substantial further resources will be needed for all three initiatives. But the excellent news is that interest in taxonomy is reawakening, and the taxonomic and informatics communities are working together to accomplish much more than either could achieve separately.

Frank A. Bisby ${ }^{\star}$, Junko Shimura†, Michael Ruggiero $\ddagger$, James Edwards§, Christoph Haeuser||

*Species 2000 Secretariat, Reading, UK $†$ Species 2000 Asia-Oceania Secretariat and GTI National Focal Point of Japan, Tsukuba, Japan \$ITIS Secretariat, Washington DC, USA §GBIF Secretariat, Copenhagen, Denmark ||Chairman of the GBIF Governing Board and GTI National Focal Point for Germany, Stuttgart, Germany Address for correspondence: F.A.B., Centre for Plant Diversity and Systematics, School of Plant Sciences, University of Reading, Reading RG6 6AS, UK f.a.bisby@sp2000.org

\section{Strong case for neutrons}

Sir - On 18 July you reported (Nature 418, $262 ; 2002)$ a review of major facilities and an assessment of the European Spallation Source (ESS) by Germany's science council, the Wissenschaftsrat. As your report says: "The council endorsed an assessment of the ESS by a subcommittee ... that the demand for neutrons does not justify the estimated 1.4-billion-euro (US\$1.4-billion) total investment in an advanced neutron source." This assessment reflected neither the consensus view of the subcommittee nor the opinion of most members. As members of this subcommittee, we have written to the chair of the Wissenschaftsrat with our concerns, paraphrased below.

"Contrary to the impression given by the draft subcommittee report presented to the Wissenschaftsrat, most of the committee judged the scientific case for the ESS to be very strong. The research opportunities for many fields of applied and fundamental science, such as solid state physics/chemistry, particle physics, biology and engineering, were seen as novel and outstanding. Critical remarks by some members were confined to the area of polymer physics/chemistry.

"Further, contrary to the summary of our deliberations in the Wissenschaftsrat's report, we do not see reason for concern about the timeliness of the scientific programme. We agree with the Japanese and US research communities, who have successfully sought long-term funding for high-intensity spallation sources, that experiments with neutrons will play a leading role across the sciences for the foreseeable future.

"The Wissenschaftsrat's statement on the cost of neutrons relative to other techniques is not based on input from us and we do not believe it would be supported by a comprehensive analysis."

\section{B. Keimer}

Max Planck Institute for Solid State Research, 70569 Stuttgart, Germany

Other signatories to this letter:

H. Dosch Max Planck Institute for Metals Research,

Stuttgart, Germany

D. Dubbers University of Heidelberg, Germany

H. Fuess Technical University of Darmstadt, Germany

T. Mason Spallation Neutron Source, USA

E. Sackmann Technical University of Munich, Germany

P. Withers University of Manchester, UK 